Ana Giménez Calpe

Universität Granada, Granada

DOI: $10.19195 / 0435-5865.141 .5$

\title{
Weibliche Ohnmacht und Gewalt in Elfriede Jelineks Der Tod und das Mädchen III (Rosamunde). Performative Lektüren
}

\section{Einleitung}

Frauen, die erschossen werden, Körper, die in einem Tunnel brennen, oder Sportereignisse, die an Feldschlachten erinnern, sind nur einige der vielen Beispiele in Elfriede Jelineks Theater, die die zentrale Bedeutung von Gewalt in den Texten ausweisen. Doch nicht nur physische Gewaltereignisse werden in Jelineks Texten thematisiert; die Gewalt drückt sich darüber hinaus in der Sprache der Figuren aus. Deswegen hat Natalie Bloch zu Recht bemerkt, dass in Jelineks Texten, wie in anderen zeitgenössischen Theatertexten, die Gewalt diskursiv verhandelt wird (Bloch 2011: 15).

Diese sprachliche Gewalt lässt sich exemplarisch in dem Dramolett Rosamunde zeigen, dessen Ausgangspunkt die Hauptfigur eines eher unbekannten romantischen Schauspiels von Helmina von Chézy ist. Bereits der Titel enthält den intertextuellen Verweis auf das romantische Stück, das Jelineks Text zitiert, subvertiert und verfremdet.

Elfriede Jelinek distanziert sich in ihrer Lektüre des romantischen Textes von dem klischeehaften romantischen Diskurs, der die weibliche Protagonistin als gutherzige Frau darstellt, und formt ihre Protagonistin in eine gequälte und hasserfüllte Frau um. Wird die weibliche Figur des romantischen Textes als gutmütig dargestellt, so liefern die zwei Protagonisten in Jelineks Stücks einen dialektischen Kampf, in dem sie sich gegenseitig beleidigen und beschimpfen. Indem die weibliche Sprechinstanz als Autorin charakterisiert wird, konzentriert sich die Auseinandersetzung zwischen den Figuren auf einen ganz konkreten Streit, nämlich auf den Kampf um die Macht über die Sprache. Das Wort erweist 
sich als Mittel, an die Macht zu gelangen bzw. diese zu bewahren. Das Verhalten der Figuren bestätigt somit das Verfahren, das Jelinek im Text verwendet und mit dem sie die Beziehungen zwischen strukturellen Machtverhältnissen und Sprache entlarvt.

Bei dem Zitat des Prätextes weicht Elfriede Jelinek voll und ganz von der Originalhandlung ab. In Chézys romantischen Text ist Rosamunde die Prinzessin von Zypern. Nachdem ihre Eltern von Rosamundes Onkel Fulvio vergiftet werden, wird sie im Geheimen als Hirtin erzogen. Erst mit sechzehn Jahren erfährt sie von ihrer Pflegemutter Axa, dass sie die wahre Erbin des Thrones ist, doch ihr Onkel Fulvio, der seit dem Tod des Königspaars das Land regiert hat, will nicht auf seine Macht verzichten und versucht, Rosamunde mit einem vergifteten Brief umzubringen. Die Gutherzigkeit von Rosamunde erschüttert ihn jedoch und so berührt er absichtlich den vergifteten Brief und stirbt. Rosamunde verliebt sich unterdessen in Alfons, den Prinzen von Candia, und wird schließlich die Herrscherin des Landes. Weder von der Erzählstruktur noch von der Thematik sind Spuren in Jelineks Text zu erkennen. Doch die Vorstellung, dass die Frau nicht als legitime Herrscherin anerkannt wird, wird im Dramolett der Ausgangspunkt, um die Definition der weiblichen Ohnmacht zu thematisieren.

Susann Neuenfeldt verweist in ihrem Beitrag „Tödliche Perspektiven“ auf die Nachwirkungen struktureller und genderspezifischer Gewalt, unter denen die weibliche Sprechinstanz Rosamunde leidet (Neuenfeldt 2005: 156). In ihrer Analyse untersucht sie nicht nur das Dramolett Rosamunde, sondern alle Prinzessinendramen, einen Zyklus von fünf kurzen Theaterstücken, den Jelinek 2003 unter dem Titel Der Tod und das Mädchen I-V veröffentlichte. ${ }^{1}$ Die motivisch miteinander verbundenen Dramen können als individuelle Werke gelesen werden - und so werden sie auch oft interpretiert. Gleichzeitig bilden die Stücke ein zusammenhängendes Ganzes, in dem die einzelnen Interpretationen der Texte sich gegenseitig bereichern. So unterschiedlich die Struktur und die Referenzen der Stücke sind, verfügen alle Texte des Zyklus über einen roten Faden: Wie der Untertitel des Buches, Prinzessinnendramen, ankündigt, wird von Jelinek in ihren Stücken ein bestimmter Frauentypus, jener der Prinzessin, in all seinen Facetten untersucht. Die Autorin problematisiert die Stilisierung der Prinzessin, die sowohl von der hohen, wie auch von der populären Kultur tradiert wird, und betont bzw. enthüllt den Mangel an Macht, der bei der Prinzessin als Frauenideal implizit ist.

${ }^{1}$ Die ersten drei Dramen waren zwischen 1998 und 2002 zum ersten Mal veröffentlicht worden. So wurde das erste Drama Der Tod und das Mädchen I in dem Band Macht nichts. Eine kleine Trilogie des Todes (1999) herausgegeben. Ein Jahr später erschien Der Tod und das Mädchen II in Das Lebewohl (2000) und 2002 wurde Rosamunde, das dritte Dramolett des Zyklus, als Teil des Buches In den Alpen. Drei Dramen (2002) veröffentlicht. Inzwischen schrieb Jelinek noch die zwei letzten Dramolette, die sie auf ihrer Webseite veröffentlichte, bis erst 2003 die fünf Stücke sowie der Essay Die Prinzessin in der Unterwelt (Statt eines Nachworts) Eingang in einen Einzelband fanden. Vgl. Arteel (2013: 174-185). 
Neben der klischeehaften Definition der weiblichen Ohnmacht wird in Jelineks Text auch aufgezeigt, wie die Unterdrückung der Frau mit Gewalt installiert wird. Indem die Frauenfiguren der Dramoletten aus der Perspektive der Toten sprechen und handeln, werden die für die Frau tödlichen Konsequenzen des patriarchalen Systems enthüllt. Zwar ist es richtig, dass das Sprechen der weiblichen Protagonistinnen aus diesem „U-Topos“ (Neuenfeldt 2005: 148), in dem sie sich als tote Frauen befinden, von einer strukturellen, gesellschaftlichen und physischen Gewalt gegen sie zeugt. Doch ebenso sind die Unterdrückungsmechanismen in der Sprache der Figuren verortet, wie ich im Folgenden ausführlich bei der Analyse des Dramoletts Rosamunde aufzeigen werde. Das Ziel dieser Arbeit ist, die Strategien des Textes zu untersuchen, mit denen es Jelinek gelingt, die implizite und strukturelle Gewalt gegen die Frau sichtbar zu machen. Dafür bietet sich die Theorie der Performativität von Judith Butler besonders gut an. Vor allem ihre Untersuchung von den Widerstandsmöglichkeiten gegen die verletzende Äußerungen sind für diese Arbeit von Bedeutung. Argumentiert die Philosophin Judith Butler in ihrem Text Excitable Speech: A Politics of the Performative, dass eine Reartikulation und eine Resignifizierung der verbalen Gewalt über die Konzepte von Wiederholung und Zitation möglich seien (Butler: 1997), so zeigt die Verwendung von aggressiver, verletzender Sprache in Rosamunde vor allem, dass sie ein kritisches Potential birgt, mit dem Jelinek die gesellschaftlichen, gewaltigen Machtverhältnisse sichtbar macht und dadurch auflöst.

\section{Die weibliche Stimme: Die performative Konstruktion der ohnmächtigen Prinzessin}

Der Tod und das Mädchen III fängt mit einer nicht identifizierten Stimme an, die, wie auch die Protagonistinnen der andren Dramoletten, eine eigene Stimme fordert, um die beschränkte Existenz zu reflektieren und in Frage zu stellen (Vgl. Jirku 2008: 10). Nach einem kurzen Intermezzo, wird die Figurenkonstellation von Chézys Drama auf ein aufeinandertreffendes Paar reduziert. Bei Jelinek treten Rosamunde und Fulvio als verfeindete Figuren auf, zwischen denen sich ein wahres Duell entwickelt. Hass und Gewalt prägen die dialektische Auseinandersetzung zwischen den Figuren, wie in den Regieanweisungen beschrieben wird: „Die zwei machen sich fertig, aber echt!“ (Jelinek 2012: 49) Wie auch in dem ersten Prinzessinnendrama Schneewittchen verschwindet in Jelineks Version die positive Figur des Prinzen, der in den Originaltexten die Funktion des Retters verkörpert. Nur der Antagonist Fulvio begleitet Rosamunde. Ihre Auseinandersetzung versinnbildlicht den allgemeinen Kampf zwischen Frau und Mann, der im Stück sich auf den konkreten Kampf um die Macht über die Sprache konzentriert. 
Doch in wie weit distanzieren sich die Figuren in Jelineks Text von dem Figurenpersonal des Prätextes? Und welche Beziehung gibt es zwischen der Stimme des Monologs und den Figuren, die im Dialog sich dialektisch konfrontieren? Die Frage ,wer spricht?“", die im zeitgenösischen postdramatischen Theater sich anders als im dramatischen Theater beantworten lässt, scheint hier, wie in anderen Texten Jelineks, auch von Bedeutung zu sein. ${ }^{2}$ Stellt sich die Problematik in Jelineks Werk mit neuer Dringlichkeit im Monolog, so scheint diese im Dialog beantwortet zu werden. Die weibliche Stimme im Monolog bleibt namenlos und erst im Dialogpart werden ihr Name - Rosamunde - und Körper zuteil. ${ }^{3}$ Stimme und Figur verfügen über ähnliche Eigenschaften, die sie in Verbindung bringen: Beide stellen sich als Autorinnen dar und werden von äußerlichen Instanzen bedroht. Doch bevor die weibliche Figur Rosamunde sich mit Fulvio dialektisch auseinandersetzt, bekommt sie die Möglichkeit, die Version ihrer Geschichte zu erzählen. Körperlos nimmt die im Monolog dargestellte Instanz eine Stimme in Anspruch, mit der sie ihre Selbstdarstellung vermitteln kann. Sie hat weder eine Form, noch ist sie gesellschaftlich definiert, denn diese Eigenschaften bekommt sie erst, wenn sie als Figur ihrem männlichen Konterpart, Fulvio, im Dialog gegenübertritt.

In ihrem Versuch, ihr Ich zu definieren, weist die Stimme des Monologs auf eine Vielfalt von Bedeutungen hin. Sie assoziiert ihre Identität abwechselnd mit verschiedenen Bildern, die im Laufe des Textes variieren (Vgl. Lücke 2005: 128). In der aquatischen Umgebung, in der sich Rosamunde befindet, scheint die Durchlässigkeit der Bilder, mit denen sie ihr Dasein beschreibt, ein besonderes Kennzeichen zu sein. Somit stellt das Drama die traditionellen Normierungen und Zuschreibungen in Frage. Rosamundes Identität wird von keinen festen und unveränderbaren Kategorien gebildet, denn alle Bilder sind ,aufgeweicht“ (Vgl. Lücke 2005: 131) und flüssig gemacht. So stellt sie sich mehrmals als Ertrinkende dar, aber auch als eine Autofahrerin, die einen Unfall erleidet, als ein ,weinend[es] Kind“ (Jelinek 2012: 47), „ein schüchtern[es] Reh“ (Jelinek 2012: 47) oder ein entmastetes Schiff, das ,seit längerem [...] auf dem Meer“ (Jelinek 2012: 45) irrt. In diesem Prozess der Selbstbestimmung greift die Stimme auf unterschiedliche gesellschaftliche Klischees über die Frau zurück, die in ihrem Diskurs wiederholt werden. Viele dieser Bilder entnimmt Elfriede Jelinek Chézys romantischem Schauspiel Rosamunde. Damit werden die klischeehaften Zuschreibungen Chézys

${ }^{2} \mathrm{Zu}$ der Frage nach dem Ursprung der Rede im postdramatischen theatralen Sprechen hat sich Evelyn Annuß wie folgt geäußert: „An die Stelle der Phänomenalisierung personaler Referenz durch den Körper auf der Bühne, die das dramatische Theater als Identifikationsangebot liefert, tritt die offene Frage, wer $d a$ in wesen Namen und zu welchem Zweck spricht.“ (Annuß 2005: 29).

${ }^{3}$ In der Sekundärliteratur wird diese Lektüre, nach der die Stimme des Monologs der weiblichen Figur des Dialogs gehört, von den meisten ForscherInnen vertreten. Bärbel Lücke, die in ihrer Analyse auch die Stimme mit der Figur Rosamunde identifiziert, betont jedoch, dass die Frage im Text offen bleibt. Vgl. Lücke (2005: 128). 
Textes enthüllt, die in ihrer Konstruierheit ausgestellt und durch die verfremdete Wiederholung aufgelöst werden.

Die Vielfalt der evozierten Frauenbilder, die ständig gewechselt werden, stellen die weibliche Identität Rosamundes als performative Konstruktion dar. Ihr Dasein entwirft sie als Ergebnis diverser etablierter Weiblichkeitsbilder, die sie in ihrem Diskurs wiederholt. Dazu spielt die Struktur des Dramoletts eine wichtige Rolle, wie im Folgenden argumentiert werden soll. Gerade durch die Struktur problematisiert Jelineks literarischer Text die Kategorie der Identität als feststehender Bezug, so wie die Philosophin Judith Butler auf theoretischer Ebene gemacht hat. Jelineks Dramolett exemplifiziert Vorstellungen feministisch-konstruktivistischer Positionen, die den Subjektbegriff als autonome Kategorie in Frage stellen. Vertritt Judith Butler in ihrem erfolgreichen Buch Gender Trouble (1990), dass die Identität ein Produkt von Diskursen und nicht eine Darstellung einer diesen Diskursen vorgänglichen Realität sei, so wird der Prozess des Subjektwerdens Rosamundes im Stück auf eine sehr graphische Art und Weise aufgezeigt. Wie ich schon beschrieben habe, folgt der Dialog zwischen zwei Figuren einem anfänglichen Monolog, von einer nicht identifizierten Stimme ausgesprochen. Bevor die Figur Rosamunde im Dialog als solche erscheinen kann, wird ihre Identität von einer Stimme konstituiert, die ununterbrochen unterschiedliche etablierte Diskurse wiederholt. Ihre Identität kann die Figur nicht frei wählen, denn diese wird, wie Judith Butler darlegt, mittels verschiedener sozialer Stereotypen reguliert, die ständig wiederholt und zitiert werden (Vgl. Butler 1990). Über die sich wiederholenden (und intertextuellen) Diskurse entsteht Rosamundes Identität. Wie die anderen Figuren der Prinzessinendramen, bedient sich Rosamunde eines Repertoires gesellschaftlicher Klischees, um ihre Identität performativ herzustellen (Vgl. Borowski und Sugiera 2008: 247). Doch der Diskurs geht im Sinne Butlers dem Erwerb einer eigenen Identität voraus. Der Text zerstört die Vorstellung eines prädiskursiven Subjekts, denn die Protagonistin entsteht als Subjekt erst im zweiten Teil des Textes, nachdem die Stimme im Monolog ihre Identität durch die Wiederholung von konventionellen Weiblichkeitsdefinitionen herstellt. So bekommt die Protagonistin ihre Identität erst im Dialog, als sie endlich benannt wird und als Rosamunde auftritt.

\section{Die Tradition revidieren: Die Wassermetaphorik und der Metadiskurs über die weibliche Autorschaft}

Den Motivkomplex Weiblichkeit und Tod, dem Jelinek aus unterschiedlichen Perspektiven in den fünf Dramoletten nachgeht, bearbeitet die Autorin in Rosamunde mittels der Darstellung eines konkreten weiblichen Todes, und zwar des Todes im Wasser. Jelinek knüpft auf diese Weise an die Ikone der Wasserleiche an, die eine 
lange Tradition in der westlichen Literatur- und Kulturgeschichte hat, um auf das traditionelle Topos zu verweisen und es kritisch zu beleuchten.

Zum Motiv des Wassertodes in der Literaur hat Anna Maria Stuby hingewiesen, dass in allen Epochen die Ästhetisierung des Todes im Wasser sich überwiegend auf Frauen bezieht (Vgl. Stuby 1992: 183 ff). Der Tod durch Ertrinken wird als ein sanftes, friedliches und fast angenehmes Sterben dargestellt, aus dem sich eine schöne Wasserleiche ergibt, wie es der Fall in der Darstellung des unglücklichen Endes von Ophelia in Shakespeares Hamlet ist. Die Stilisierung des Todes Ophelias bildet den Anfang einer langen Tradition zahlreicher Kunstdarstellungen, in denen die Faszination für die weibliche Wasserleiche weiter entwickelt wird. Die Ophelia (1851/1852) von John Everett Millais oder das Gedicht Ophélie (1870) von Arthur Rimbaud sind einige Beispiele aus dem 19. Jahrhundert, die die damalige Ophelia-Begeisterung belegen. Das transgredierende und zerstörerische Potential der Sirene, die während des Biedermeiers zugunsten von einer verbürgerlichten Anpassung des mythischen Wesens geopfert wird, verschwindet voll und ganz mit der literarischen und künstlerischen Bearbeitung des Ophelia-Motivs. In der „schönen Leiche“ (Bronfen 1994), die als harmonisches Bild dem Blick des Beobachters erhalten bleibt, ist keine Spur von der gefährlichen erotischen Anziehung der Sirene zurück geblieben. Auch das Stummwerden der Sirene, ein literarisch rekurrentes Motiv, wird in der Form der Wasserleiche vollendet. Wie die Wasserfrau in Hans Christian Andersens Märchen Die kleine Seejungfrau (1837), die ihre Stimme gegen menschliche Beine austauscht, ist die weibliche Leiche im Tode ihrer Stimme beraubt. Die weibliche Leiche im Wasser, die weder über Leben noch über Stimme verfügt, stellt auf diese Weise keine Gefahr mehr für den männlichen Betrachter dar.

Diese literarische Tradition wird in Rosamunde evoziert und problematisiert, indem die Protagonistin sich als Ertrinkende beschreibt, sich jedoch im Gegensatz zu Andersens Seejungfrau weigert, ihr sprachliches Potential für immer zu verlieren. Bevor ihre Stimme verstummt, ergreift sie noch das Wort, um über den Zustand des eigenen Todes zu reflektieren: „Wie kalt das ist, kein Mondlicht so friedlich, kein Traum, der mich täuscht. Keine lichte Welt, die mich umblüht, kein süßer Blick, der mich umstrahlt, nicht einmal der Zigarettenzünder, [...].“ (Jelinek 2012: 47) Rosamundes Beschreibung von ihrem Ertrinken entfernt sich von der ästhetisierten Darstellung des weiblichen Todes, die in der Literatur- und Kulturtradition geschildert wird. Mit ihren Worten weigert sie sich, als lebloses Kunstobjekt charakterisiert zu werden, und betont ihre Gefühle von Einsamkeit und Kälte. Aus der Perspektive der sterbenden Frau wird die Version des friedlichen Sterbens hinterfragt und als stilisierte Konstruktion offen gelegt.

Der Hinweis auf den Zigarettenzünder, dessen Licht auch nicht der Dunkelheit von Rosamundes Umgebung entgegenwirken kann, versetzt die Handlung des Stückes in die Aktualität. Diese zeitliche Verschiebung trägt zum einen zur 
Betonung des Weiterlebens der festgeschriebenen Rollenbilder bei, zum anderen betont es den Charakter ihrer Konstruktion.

Noch eine weitere Referenz, womit die Handlung des Textes in die Gegenwart versetzt wird, ist im Text zu finden: Die Stimme des Monologs, die sich als ertrinkende Frau ausgibt, stellt sich paradoxerweise auch als Opfer eines Autounfalls dar: „Wogen, die mir Liebe spiegeln, wenigstens die schaun mich an, hab ich zumindest geglaubt, doch es war ein Unfall mit dem Wagen. Nicht einmal des Bremslichts vom Vordermann konnt ich mich recht erfreuen." (Jelinek 2012: 46) So wie die Bilder, mit denen die Sprecherin des Monologs ihre Identität assoziiert, im Laufe des Textes variieren, spricht sie auch nicht von einem einzigen Unfall, der ihr Leben gefährdet: „Ja, genau so habs ich persönlich erfahren, mit völlig abgefahrnen Reifen, und jetzt dieser schwere, vollkommene unvermeidliche Unfall, geschieht mir ganz recht. Jeder hätte das sofort gesehen, daß dieses Wasser nur drauf gewartet hat, mich umzubringen." (Jelinek 2012: 46) Indem das Wasserunglück nicht mehr von einem entmythologisierten und brutalen Autounfall zu unterscheiden ist, wird das idealisierte künstlerische Motiv des weiblichen Ertrinkens destabilisiert. Darüber hinaus wird die ausgeübte Gewalt gegen die Frau nicht mehr als Naturereignis dargestellt, sondern wird im Text durch menschliches Handeln hervorgerufen. Der Konstruktionscharakter des literarischen und künstlerischen Motives wird auf diese Weise offen gelegt.

In der Beschreibung ihrer komplizierten Lage versteckt Rosamunde nicht die physische Gewalt, unter der sie leiden muss: „Jawohl, jetzt kommts, hier, plötzlich, das Blinklicht von Fluten, die nicht für mich gebremst haben, obwohl sie es sogar für Tiere tun. Und da bohrt sich doch glatt dieser Zinken von einer Flut in meinen Kühlergrill.“ (Jelinek 2012: 43-44) In der Aussage Rosamundes identifiziert Britta Kallin den phallischen Charakter des Zinkens, der in den „Kühlergrill“ (Jelinek 2012: 44) der weiblichen Stimme bohrt (Kallin 2007 [o. S.]). Die Stimme, die in diesem Fall sich als Autofahrerin ausweist, leidet unter der physischen Gewalt, die das andere Auto hervorbringt. Der Kühlergrill, der metonymisch für den weiblichen Körper steht, wird gewaltsam und brutal vom phallischen Objekt durchbohrt. Wird in der literarischen und bildkünstlerischen Tradition die Wasserleiche als Ergebnis eines sanften und ruhigen Prozesses dargestellt, so wird die Gewalt gegen die weibliche Protagonistin in Jelineks Drama sichtbar gemacht. Im beschriebenen Szenario des weiblichen Unfalls sind keine ruhigen Gewässer zu finden, sondern „Fluten“( Jelinek 2012: 43), die in Form des „Blinklichts“" (Jelinek 2012: 43) gewaltig über das weibliche Opfer hereinbrechen. Der Text zerstört die künstlerischen Fantasien, die den toten weiblichen Körper als faszinierend und ästhetisch produktiv begreifen, und macht hingegen die verborgene Gewalt gegen die Frau sichtbar.

Neben der körperlichen Gewalt, erfährt Rosamunde noch eine andere Art von Gewalt, nämlich die der Ausgrenzung und der gesellschaftlichen Nicht-An- 
erkennung. Anzumerken ist, dass Jelinek bei ihrer Adaptation auf ein Thema verweist, das nicht im romantischen Prätext behandelt wurde, und zwar die weibliche Schöpferkraft. Wird die Ohnmacht der Frau in den Prinzessinnendramen in verschiedenen Facetten ästhetisch und kritisch verhandelt, wofür die Theatertexte populärer lebendiger oder fiktiver Frauenfiguren der westlichen Kultur, wie Jackie Kennedy oder der Märchenprotagonistinnen Schneewittchen und Dornröschen, sich bedienen, so ist die Frage des Schreibens in Rosamunde von zentraler Bedeutung. Die weibliche Figur wird in diesem Sinne als eine erfolglose Autorin charakterisiert, die in einer männlich bestimmten Kultur von dem literarischen Kanon ausgeschlossen wird. Dafür entfaltet die Autorin ein weit gefächertes intertextuelles Spiel, in dem sie auf das Drama Rosamunde von Chézy Bezug nimmt. Das Drama, das zu seiner Zeit ein Misserfolg war, überlebte nur in der Musik von Franz Schubert, der das Libretto 1830 vertonte. Während Helmina von Chézys Drama in Vergessenheit geriet und sogar bis 1996 als vermisst galt, wurde die Vertonung von Schubert ein bekanntes Musikstück, das dem Kanon angehört.

Rosamunde - sei es in der Form einer Stimme des Monologs oder als Figur, die ihrem männlichen Kontrahenten entgegentritt, - lässt in ihren Bemühungen nie nach, das Wort zu ergreifen, um ihre persönliche Version über ihre Existenz zu vermitteln. Dafür gibt sie sich nicht nur mit der gesprochenen Sprache zufrieden sondern greift auch auf die Schrift zurück. Dies ist insofern von Bedeutung, da Rosamunde mit dieser Aktivität die traditionellen Grenzen der Geschlechterdichotomie überschreitet. Entgegen der hegemonialen Diskurse traut sich die Protagonistin, Subjekt sein zu wollen und zwar als Schöpferin, als Produzentin.

Doch Rosamundes Aneignung der Sprache bringt, wie sie selbst auch anerkennt, wenige Veränderungen mit sich. Ihr Versuch, den männlichen Logos für sich in Anspruch zu nehmen, hat keine realen Auswirkungen, denn ihre Stimme - und hier sind gerade ihre schriftlichen Texte eingeschlossen - wird nicht gehört. Die Macht, die die Stimme für sich mittels der Sprache und der Schrift wünscht, wird wegen des Mangels an Anerkennung nicht real. Sie wird eine Königin ohne Reich, eine Figur, die in ihrer märchenhaften Version ein reales Problem andeutet: „[...] ich schreib und schreib, die Königin der Welt bin ich, nur sieht mich wieder einmal keiner.“ (Jelinek 2012: 44)

Jelinek greift die Problematik der weiblichen Schöpferkraft auf, die in einer männlich bestimmten Kultur von dem literarischen Kanon ausgeschlossen wird, und macht das Leiden sichtbar, das das Gefühl des Nicht-Gehörtwerdens bei vielen Autorinnen auslöste. ${ }^{4}$ Die Auseinandersetzung mit dem romantischen Text

${ }^{4}$ Dieses Thema schreibt Jelinek in Die Wand, dem fünften Dramolett der Prinzessinnendramen, weiter. Hier verweist sie offensichtlicher auf die Verschränkung von weiblicher Autorschaft und Suizid, ein Thema, das in Rosamunde durch die Assoziationen an Autorinnen, die sich das Leben durch Ertrinken nahmen, wie Virginia Woolf, Alfonsina Storni oder Aglaja Verteranyi, auch in Erinnerung gerufen wird. 
Rosamunde findet hier im doppelten Sinn statt, sowohl auf der Autoren- als auch auf der Figurenebene. Einerseits nimmt Jelinek Bezug auf eine Autorin, die keinen Erfolg als Schriftstellerin erlebte, auch wenn sie sich mit ihrem Text Rosamunde sowohl an die gesellschaftlichen als auch an die literarischen Konventionen ihrer Zeit anpasste. Anderseits wird die Ausschließung weiblicher Autorinnen vom literarischen Kanon auf der Figurenebene thematisiert, indem Rosamunde sich als gescheiterte Autorin charakterisiert. Die fehlende Anerkennung Rosamundes und ihrer Arbeit in Jelineks Stück verbindet die Stimme mit Chézys Rosamunde. Diese ist eine junge Frau, die isoliert und versteckt erzogen wird, auch wenn sie, als Tochter des verstorbenen Königs von Zypern, die wahre Erbin des Thrones ist. Wird das Unrecht im originalen Drama behoben, so wird Jelineks Figur, die sich nicht als Prinzessin sondern als Königin ausgibt, zu ihrem Bedauern nie zur Kenntnis genommen. Nur von Einsamkeit und Drohung wird ihre Existenz bestimmt:

[...] ich schreib und schreib, die Königin der Welt bin ich, nur sieht mich wieder einmal keiner. Ich krieg schon keine Luft mehr, bange Träume schrecken mich: [...] Wo ich so lang gesucht habe, in der Welt herumgeirrt, ohne meinen Schreibtisch zu verlassen, so lang und sowieso allein, [...] Doch einsam werd ich sterben. (Jelinek 2012: 44-46)

Rosamundes Suche am Arbeitstisch stellt sie als ein vergeblicher Akt dar. Mit ihrem Schreiben kann sie die eigenen Fragen nicht beantworten; das Rätsel des Lebens wird damit nicht gelöst. In Verbindung mit dem romantischen Ideal des lebenslangen Irrens und Suchens um die Welt wirkt der Parallelismus zwischen dem Herumirren und der intellektuellen Arbeit am Schreibtisch paradox (Vgl. Kallin 2007 [o. S.]). Im Kontext des 21. Jahrhunderts lässt sich die Stelle auch so lesen, dass die Stimme im Internet sucht, ohne sich von ihrem Schreibtisch fort zu bewegen. Diese Lektüre hebt nicht nur das Paradox auf, sondern legt noch mehr Nachdruck auf die Zurückgezogenheit der Autorin, die keinen Kontakt mit der Außenwelt hat. Die Stimme bleibt allein, zurückgeworfen und erweist sich als unfähig, Antworten auf ihre Fragen zu finden. Ihr einsamer Tod versinnbildlicht ihre Unmöglichkeit, von den LeserInnen wahrgenommen zu werden. Ihre potentiale Macht als Königin ist nur eine Illusion, denn diese kann nicht real wirken, solange sie nicht von den Anderen, wie es der Fall Rosamundes in Chézys Stück ist, wahrgenommen und akzeptiert wird. ${ }^{5}$

${ }^{5}$ Indem Jelinek die Thematik der weiblichen Autorschaft auf diese Weise thematisiert, parodiert sie ihre eigene Lebenssituation als Schriftstellerin. Als die Autorin in einem Interview über ihre Beziehung mit der ertrinkenden Dichterin Rosamunde ihres Textes gefragt wurde, antwortete sie: "Das ist ja einer der autobiographischen Texte, die ich je geschrieben habe, der Prolog zumindest. Da thematisiere ich mich als alternde Frau, die sich diese Schöpferkraft zumißt und damit voll einfährt. Ins Wasser.“ Jelinek (2005: 48-49). 


\section{Die diskursive Gewalt als kritische Bearbeitung}

Stellt sich Rosamunde im Monolog als Opfer einer schwer identifizierbaren Gewalt dar, die in Form einer "Wassermasse“ (Jelinek 2012: 46) erscheint, so tritt Fulvio im Dialog als Figur auf, die so auf konkretere Weise die Gewalt gegen die Frau verkörpert. Die Gewalt im Theatertext wird jedoch nicht als ein physischer Kampf zwischen den Figuren repräsentiert, sondern in der Sprache der Figuren ausgedrückt. Wie in anderen Texten der Autorin, wird der Fokus auf die sprachliche Verhandlung von Gewalt verschoben, die ästhetisch im Text untersucht wird, um konkrete Machtbeziehungen, die symbolische und kulturelle Gewalt ausüben, sichtbar zu machen.

Die dialektische Auseinandersetzung zwischen den Figuren konzentriert sich in Rosamunde auf eine ganz konkrete Weise auf den Kampf um die Kontrolle über die Sprache. Die Frage des Schreibens wird, wie Bärbel Lücke feststellt, „,in die der Geschlechterdifferenz eingeschrieben“ (Vgl. Lücke 2005: 130). Vom Paradigma der Geschlechterdifferenz überzeugt, kritisiert Fulvio mit besonderer Heftigkeit Rosamundes schriftliche Aktivität. Die weibliche Aneignung des als männlich kodierten Logos findet er lächerlich und unangemessen:

Ich nehm dich so lang, bis ich dich wiedergewinnen kann aus dir selbst. Dann laß ich deine letzte Hülle fallen. Was beklagst du dich? Die letzten Hüllen hast du doch vorhin selber fallen lassen! In deinem Schreiben, wie du sagst. [...] Aber schau, was du für deine letzten Hüllen gehalten hast, das war bloß die Kapsel von deinem Kugelschreiber! Kein Bedarf! (Jelinek 2012: 49)

Die sexuelle Konnotation der ersten Sätze, mit denen der männliche Protagonist Fulvio sich selbst die sexuelle aktive Rolle zuschreibt, wird im Text mit dem Prozess des Schreibens verbunden. Doch nach Fulvio ist das Ausziehen für die Frau nur im wörtlichen Sinn möglich. Kann sie sich von ihren „Hülle[n]“(Jelinek, 2012: 49), von ihren Kleidern befreien, um vom Mann genommen zu werden, so scheitert sie daran, sich von ihren „Hülle[n]“ (Jelinek, 2012: 49) im metaphorischen Sinn zu lösen. Rosamundes Absicht, mit ihrer schriftlichen, kreativen Tätigkeit, ihr Inneres ohne ornamentale „Hülle“ (Jelinek, 2012: 49) aufzuzeigen, wird von Fulvio als bloße Illusion entlarvt. Die kreative Aktivität der weiblichen Autorin wird somit von der männlichen Figur parodiert und die weibliche Existenz auf den Status eines unterwürfigen und begehrten Objekts beschränkt.

Die diskursive Gewalt Fulvios gipfelt in der Ankündigung, er werde Rosamunde ihre Zunge abschneiden: „Ein Fest ist immer da, das uns ruft. So, Rosamunde. Danach ist ihre Zunge die Rede nicht wert, die sie so lose geführt hat. Alles nur Show. Die Zunge schneide ich dir ab, und wo ist jetzt das Wort? Siehst du, weg ist es!“ (Jelinek 2012: 53) Die Rede der Frau betrachtet er nur als endlosen Redeschwall, der von Banalität und Bedeutungslosigkeit geprägt ist. In diesem Sinn verwendet er kurz danach den Ausdruck „Mädchengeschwätz“ (Jelinek 
2012: 53), um sich abwertend auf ein unbedeutendes Gespräch zu beziehen. Die Rede Rosamundes, als konkretes Anzeichen der weiblichen Sprache, wird auf eine ähnliche Art und Weise charakterisiert: Ihre Zunge, die metonymisch für den Akt des Sprechens steht, bewegt Rosamunde pausenlos, doch daraus entsteht so Fulvio nichts Wertvolles, denn alles ist „nur Show“ (Jelinek 2012: 53).

Die Beleidigung und die Missachtung der weiblichen Sprache münden jedoch in Fulvios Redepart in eine explizite Drohung, mit der Fulvio seinen Wunsch ausdrückt, Rosamundes Zunge abzuschneiden. Anhand einer Metonymisierung stellt der männliche Protagonist die Vollendung eines Prozesses dar, mit dem die Frau zum Schweigen verpflichtet wird. Mit bitterer Ironie stellt der Text die Unverzüglichkeit der performativen Wirkungen der Drohung dar. Sobald Fulvio den Satz ausspricht, verliert Rosamunde die Fähigkeit des Sprechens: „Die Zunge schneide ich dir ab, und wo ist jetzt das Wort? Siehst du, weg ist es!“ (Jelinek 2012: 53) Die Vorstellung, dass Sprechen verwundet, tritt hier im wörtlichen Sinne in Kraft: Fulvios Drohung vermittelt nicht nur ein Hassgefühl, sondern ist ein verletzender Akt per se (Vgl. Austin 1975). Der Sprechakt erzeugt das, was er benennt; er performiert den Schnitt, die Verstümmelung. Fulvios übertriebene Selbstsicherheit ist seine sonderbare Reaktion auf die weibliche Aneignung des männlichen Logos. Indem er die unverzüglichen performativen Wirkungen seiner Rede beschreibt, inszeniert er sich wieder als privilegierten Sprecher, der aus seiner Machtposition mit seinen Worten die Sprache Rosamundes beseitigen kann.

Fulvios aggressive Sprache wäre ein Beispiel dafür, was unterschiedliche Studien aus der Linguistik oder der feministischen Theorie als gewaltsame Sprechhandlungen identifiziert haben. Nach diesen Ansätzen, können bestimmte sprachliche Akte verletzen. In diesem Sinne beschreiben Formulierungen, denen die Fähigkeit des Verletzens zugeschrieben wird, nicht Verletzungen bzw. lösen diese auch nicht aus; vielmehr wird die Verletzung mit ihnen konstituiert. Die Philosophin Judith Butler geht jedoch in Excitable Speech: A Politics of the Performative einen Schritt weiter und behauptet, dass es die Möglichkeit gibt, die durch dieses Sprechen hervorgerufenen Effekte zu stören und zu unterlaufen (Vgl. Butler 1997). Dafür geht Butler davon aus, dass das Sprechen sich in gewissem Sinne der absoluten Kontrolle entzieht, so dass nicht jeder Sprechakt gelingen muss. Dieser Ansatz, der Widerstandsmöglichkeiten gegen hate speech sucht, ermöglicht eine neue Lektüre von Jelineks Text, denn in diesem soll die Verwendung der gewaltsamen Patriarchalsprache keine Bestätigung der patriarchalen gesellschaftlichen Strukturen ermöglichen, sondern eine kritische Lektüre, die zur weiteren Auseinandersetzung herausfordern will. In anderen Worten, in der Wiederholung von hate speech, die Fulvio ausführt, ist eine subversive Reartikulation im Sinne Butler möglich, mit der die gesellschaftliche Herrschaft gestört werden kann. 


\section{5. „Ein Licht, ein Nichts“. Eine weitere Destabilisierung des herrschenden Diskurses}

Fulvios Selbstvertrauen bzw. seine Sicherheit, dass er aus einer privilegierten Situation spricht, werden im Text als instabile Überzeugungen entlarvt, die sogar in der männlichen Rede von Ambivalenzen geprägt sind. Die konventionellen und stereotypierten Denkschemata münden bei Jelinek in dekonstruktiven und hybriden Bildern, die die zitierte Konvention auflösen (Vgl. Solibakke 2007: 253). Greift Fulvio die Metapher des Sonnenlichts auf, um sich als göttliche Instanz zu beschreiben, so zweifelt er daraufhin die eigene Aussage an: „Ich bin die Sonne, die auf dem Wasser glitzert. Mich gibt es zumindest zweimal. Ein Trick für die Lampe, die Flamme durch Spiegelung zu verdoppeln. Irgendwie stimmt das aber nicht. Alles wird schwächer." (Jelinek 2012: 50) Fluvios Zentralität, von ihm sonst immer festgelegt, ist, wie er selbst bekundet, nicht eine Charakterisierung ohne Fissuren. Die klischeehaften Zuschreibungen gelten ,irgendwie“ (Jelinek 2012: 50), trotz aller Versuche, sie weiter festzuschreiben, nicht mehr als absolute Wahrheit. Werden Begriffe wie Macht, Kraft und Göttlichkeit anhand des männlichen Identifizierens mit dem göttlichen Sonnenlicht in Erinnerung gerufen, so spricht Fulvio auch gleichzeitig von einem Prozess, mit dem alles „schwächer“ (Jelinek 2012: 50) wird.

Auch Rosamunde greift in ihrem Monolog die Metapher des Lichts auf, die im gesamten Text als Personifizierung der männlichen Göttlichkeit präsent ist: „Das Wasserunglück, auch nicht schlecht, ich seh es immer deutlicher, von Wehmut umglitzert, ach nein, das war ein Licht, ein Nichts, auf bloßem Abglanz auf dem Schaum der Wogen." (Jelinek 2012: 44) Das Licht und damit die männliche Göttlichkeit werden aber in diesem Zitat zum Nichts reduziert. Es hilft der Stimme nicht, die eigene Situation des Wasserunfalls besser zu überblicken. Im Gegensatz dazu erscheint das Licht als Illusion, als Trugbild, das die Stimme in die Irre führt. Denkt sie zunächst ihre Situation ,immer deutlicher“ (Jelinek 2012: 44) zu sehen, so entdeckt sie später ihren Fehler: Die Deutlichkeit ergibt sich nur aus dem betrügerischen Licht, das die Stimme mit dem Nichts gleichsetzt. Nicht der Frau, von Freud als Mangelwesen definiert, sondern dem Mann schreibt Rosamunde das Nichts als Eigenschaft zu.

Fulvios Selbstsicherheit, die die männliche Figur an einigen Stellen durch die Lichtmetaphorik ausdrückt, kontrastiert jedoch mit dem abwertenden Ton, den die Stimme des Dialogs in Bezug auf das Licht, das metaphorisch für die männliche Existenz Fulvios steht, verwendet. Stellt Fulvio fest, er existiere und zwar „zumindest zweimal“" (Jelinek 2012: 50), weil er als Abglanz auf dem Wasser ,glitzert“ (Jelinek 2012: 50), so beschreibt die Stimme das Licht als ,bloße[n] Abglanz auf dem Schaum der Wogen“ (Jelinek 2012: 44). Fulvio und die Sprecherin des Monologs verwenden fast die gleichen Referenzen, doch sie distanzieren sich bei 
ihren Charakterisierungen voneinander. Die männliche Zentralität gerät im Text durch die widersprüchlichen Beschreibungen ins Schwanken. Stellt das Stück das ungleiche Machtverhältnis zwischen den Geschlechtern dar, so hält es gleichzeitig den hegemonialen Diskurs der Geschlechterdifferenz, der für die Ungleichheit der Machtausübung verantwortlich ist, in der Schwebe.

Die hierarchische Struktur, die die Auseinandersetzung zwischen Fulvio und Rosamunde beherrscht, wird auch nicht als ein festes und unveränderbares Organisationsmodell vorgezeigt. Schreibt Fulvio sich selbst die vorherrschende Rolle zu, so gibt es auch im Gespräch gewisse Momente, in denen er von ihrer festgeschriebenen Rolle abweicht. Ebenso ist Rosamundes Verhalten wechselnd und unstabil. Oft sind Fulvios performative Akte effektiv, die dazu führen, Rosamundes gesellschaftliche Unterordnung zu verstärken. Doch auch sie spielt die Rolle der Hassenden und richtet ihre hasserfüllten Äußerungen gegen ihren männlichen Gegner Fulvio und die Patriarchalgesellschaft. Jelineks Rosamunde ist nicht mehr das gutherzige Mädchen des romantischen Werkes, das nicht hassen kann, sondern eine gequälte und rachedurstige Frau: „Ungeheuer! Unbequemer! Querdenker! [...] Ich werf und werf den $\mathrm{Ha} ß$, ich werf und werf ihn, den längst veralteten, verfallenen Haß.“ (Jelinek 2012: 54, 58) Die mythische Auffassung der romantischen Liebe wird diskreditiert. Die traditionelle Charakterisierung der Frau als zärtliches Wesen wird in Jelineks Text durchkreuzt, und die Beziehungen zwischen Männern und Frauen werden als gewalttätiger Geschlechterkampf dargestellt. Die Frau, die die eigene Diskriminierung erlebt, reagiert mit Hass und genauso agiert der Mann, der auf alle weibliche Autonomiebestrebungen mit einem Gegenschlag antwortet. Doch die Konfrontation zwischen Fulvio und Rosamunde ist kein individuelles und persönliches Problem wie im romantischen Drama von Chézy. Der Streit zwischen den Figuren in Jelineks Text stellt eine gesellschaftliche Konstellation dar, die Jelinek aus einer kritischen und demaskierenden Perspektive betrachtet.

\section{6. „Vorstellung mißlungen“. Der gemeinsame Untergang}

In Jelineks Dramolett ist es schwer von Erfolg und Misserfolg, von Siegern und Besiegten zu sprechen. Rosamundes Projekt, sich mit der eigenen Stimme zu definieren, ist nicht erfolgreich. Als schreibende Frau versucht sie das Wort zu ergreifen, um auch Wirkungen mit ihrer Stimme hervorzubringen, doch am Ende stellt sie ihren Misserfolg fest: „Meine Stimme. Meine Stimme. Meine Stimme. Meine Stimme. Sagt nichts." (Jelinek 2012: 61) Rosamundes letzte Worte, die auch die letzten des Stückes sind, kann man als die finale Feststellung des eigenen Fehlers, des eignen Misserfolgs lesen. Hat sie eine eigene Stimme beansprucht und diese als ihre eigene identifiziert, so erkennt Rosamunde diese doch wieder am Ende als fremde (Vgl. Borowski und Sugiera 2008: 251). Als Ergebnis ihres Hasses gegen Fulvio und die Patriarchalgesellschaft sowie ihrer verinnerlichten und wie- 
derholten stereotypierten Weiblichkeitsdefinitionen, bleibt Rosamundes Stimme ungehört und übt daher keinerlei Einfluss aus.

Doch Rosamundes Feststellung des eigenen literarischen Misserfolgs bringt keinen automatischen Sieg von Fulvio mit sich. Auch er, wie die bösartige Figur von Chézys Drama, erlebt seinen Abstieg in die Hölle: „ROSAMUNDE: Ich glaub, du mußt jetzt hinunter.“(Jelinek 2012: 60) und „FULVIO: [...] Schlage einen Funken in der Hölle. Völlig überflüssig. Dich liebend, konnt ich mir den Himmel nicht zurückerstürmen. Muß hinunter. [...] Nein, ich geh doch nicht, ich geh nicht, ich geh nicht! Nein, ich geh doch. Doch, doch, ich gehe!“" (Jelinek 2012: 61) Mit Fulvios Abstieg in die Hölle stellt Jelinek die Situation beider Figuren als gleichberechtigt dar, denn auch Rosamunde geht, wie sie am Ende ihres Monologs festlegt, als Ertrinkende im Wasser unter: „Ich beug mich über mich und sag was über mich und schick es ab und trag mich weg in einen tiefen Raum.“ (Jelinek 2012: 47) Mit ihrem Zitierverfahren lässt Jelinek das Ergebnis der Auseinandersetzung im Originaltext nicht ins Gegenteil umkippen. Die Lektüre, nach der die Frau als die Verliererin der Konfrontation zwischen Mann und Frau dargestellt wird, scheint in diesem Sinne eine begrenzte Interpretation. Enthüllt Jelinek im Stück die Unterdrückung der Frau sowie die fatalen Konsequenzen der patriarchalischen Literatur- und Kulturgeschichte für die schreibenden Frauen, so stellt sie auch in Rosamunde deutlicher als in anderen Prinzessinnendramen dar, dass der Hass, der die Beziehung zwischen Mann und Frau dominiert, einen vernichtenden Effekt für beide Figuren hat.

Fulvio, der in Chézys Drama sich einen Plan ausdenkt, um Rosamunde mit einem vergifteten Brief zu töten, kann diesen nicht zu Ende bringen und stirbt mit Reue, indem er den von ihm vergifteten Brief berührt:

FULVIO: O, Du himmlisch Wesen,

In deren Brust der Haß nie Wurzel schlug,

Weine um mich, ich war einst gut, die Hölle

Hat mich verlockt, Dich liebend konnt' ich mir

Dem Himmel nicht zurück erstürmen, muß

Hinunter. (Chézy 1996: 149)

Am Ende von Jelineks Stück ertönen auch ähnliche Worte, die die Autorin jedoch parodistisch verfremdet: „ROSAMUNDE: Grad schlägt der Haß in meinem Herzen Wurzeln, schon wieder, er ist zurück, ich habs ihm doch streng verboten, ach nein, er schlägt nicht andre, er schlägt mich! Ausgerechnet mich schlägt er!“" (Jelinek 2012: 60) Der vernichtende Effekt des Hasses erfährt mit Rosamundes Äußerung seine Zuspitzung. Rosamunde erlebt keine Rettung, denn als Hassende wird es auch keinen Platz für sie im Himmel geben.

Beide Figuren müssen hinunter, sei es die Hölle oder das Wasser, doch in ihrer Beziehung, die sich als Austausch von Gewalt und Gegengewalt entwickelt, ist Rosamunde die, die am meisten leidet: Nicht nur die männliche Unterdrückung ihres Schreibens, auch den eigenen Hass muss sie ertragen. Der Hass, den sie spürt, richtet sie zum Abschluss nicht nur gegen Fulvio als Vertreter der patriarchalen 
Gesellschaft, sondern zugleich auch gegen sie selbst. Jelineks Text belebt somit die verborgene patriarchale Gewaltstruktur. Diese, die weder in Chézys Originaltext noch in traditionellen literarischen Motiven wie der der Wasserleiche thematisiert wird, wird hier sichtbar gemacht. Gewalt wird jedoch nicht unter einer Perspektive betrachtet, die das Täter/Opfer-Verhältnis wiederholt. Nicht die Frage nach dem konkreten Akteur der Gewalthandlung scheint die primäre Frage im Text zu sein, vielmehr geht es in Rosamunde darum, über die strukturellen Bedingungen der Gewalt zu reflektieren und diese aufzuzeigen.

\section{Bibliographie}

\section{Primärliteratur:}

Chézy, Helmina von: Rosamunde. Tutzing 1996.

Jelinek, Elfriede: Der Tod und das Mädchen I-V: Prinzessinnendramen. Berlin 2012.

\section{Sekundärliteratur:}

Annuß, Evelyn: Elfriede Jelinek. Theater des Nachlebens. München 2005.

Arteel, Inge: Der Tod und das Mädchen I-V; Körper und Frau; Ulrike Maria Stuart; Über Tiere; Schatten (Eurydike sagt); Die Straße. Die Stadt. Der Überfall. In: Janke, Pia (Hrsg.): Jelinek Handbuch. Stuttgart 2013. S. 174-185.

Austin, John L.: How to do things with words. Cambridge 1975.

Bloch, Natalie: Legitimierte Gewalt. Zum Verhältnis von Sprache und Gewalt in Theatertexten von Elfriede Jelinek und Neil LaBute. Bielefeld 2011.

Borowski, Mateusz und Malgorzata Sugiera: Das Gedächtnis den Mythen zurückgeben oder wie macht man Prinzessinnen? In: Zittel, Clau und Marian Holona (Hrsg.): Positionen der Jelinek-Forschung. Bern 2008. S. 239-255.

Bronfen, Elisabeth: Nur über ihre Leiche. Tod, Weiblichkeit und Ästhetik. München 1994.

Butler, Judith: Gender Trouble: Feminism and the Subversion of Identity. New York 1990.

Butler, Judith: Excitable Speech: A Politics of the Performative. New York 1997.

Jelinek, Elfriede: Die Frau darf nicht begehren. In: Bühne 5, 2005. S. 48-49.

Jirku, Brigitte: Hablar desde el más allá. In: Jelinek, Elfriede. La Muerte y la doncella. I-V. Valencia 2008. S. 9-26.

Kallin, Britta: "Die Feder führ ich unermüdlich": Helmina von Chézy's Rosamunde as Intertext in Elfriede Jelinek's Der Tod und das Mädchen III: Rosamunde. In: Glossen 26, 2007. [o.S.]. http://www2.dickinson.edu/glossen/heft26/article26/kallin26.html. [Zugriff am 14.09.2015].

Lücke, Bärbel: Denkbewegungen, Schreibbewegungen - Weiblichkeits-und Männlichkeitsmythen im Spiegel abendländischer Philosophie - Eine dekonstruktivische Lektüre von Elfriede Jelineks 'Prinzessinnendramen' Der Tod und das Mädchen I-III. In: Gruber, Bettina und Heinz-Peter Preußer (Hrsg.): Weiblichkeit als politisches Programm? Sexualität, Macht und Mythos. Würzburg 2005. S. 107-136. 
Neuenfeldt, Susann: Tödliche Perspektiven. Die toten sprechenden Frauen in Elfriede Jelinek's Dramoletten Der Tod und das Mädchen I-V. In Sprachkunst 36.1, 2005. S. 147-163.

Solibakke, Karl: Serienmorde. Das Ende der Liebe, Kunst und Kultur in Elfriede Jelineks Prinzessinnendramen. In: Ahlefeld, Yvonne-Patricia (Hrsg.): Von der Liebe und anderen schrecklichen Dingen. Bielefeld 2007. S. 209-226.

Stuby, Anna Maria: Liebe, Tod und Wasserfrau. Mythen des Weiblichen in der Literatur. Opladen 1992.

\section{Abstracs}

In dem Dramolett Rosamunde, dem dritten der fünf Prinzessinendramen, die den Zyklus Der Tod und das Mädchen $I-V$ bilden, zitiert und verfremdet Elfriede Jelinek ein romantisches Stück von Helmina von Chézy, einer Autorin des 19. Jahrhunderts, die nicht sehr erfolgreich mit ihren Texten war. Dieser Artikel geht der Frage nach, wie die im Text verwendete diskursive Gewalt eine strukturelle, soziale und implizite Gewalt gegen Frauen sichtbar macht. Ausgehend von der performativen Theorie von Judith Butler, sollen die Strategien erforscht werden, mit denen der Text den etablierten Diskurs, der Weiblichkeit und Ohnmacht verbindet, destabilisiert und eine Reartikulation von hate speech möglich macht.

Schlüsselwörter: Gewalt, Performativität, Elfriede Jelinek

\section{Female powerlessness and violence in Elfriede Jelinek's Der Tod und das Mädchen III (Rosamunde). Performative readings}

In the mini-drama Rosamunde, the third of the five Prinzessinnendramen, which form the cycle Der Tod und das Mädchen I-V, Elfriede Jelinek quotes and paraphrases a romantic play written by Helmina von Chézy, a female author of the 19th century who earned little fame with her writings. This article explores the use of discursive violence to demonstrate that its use in the text uncovers social, structural and implicit violence against women. Based on Judith Butler's idea of performativity, the paper examines the way Jelinek's text destabilizes the standardized role models, which connect femininity with powerlessness, and further questions and disturbs the use of hate speech.

Keywords: violence, performativity, Elfriede Jelinek

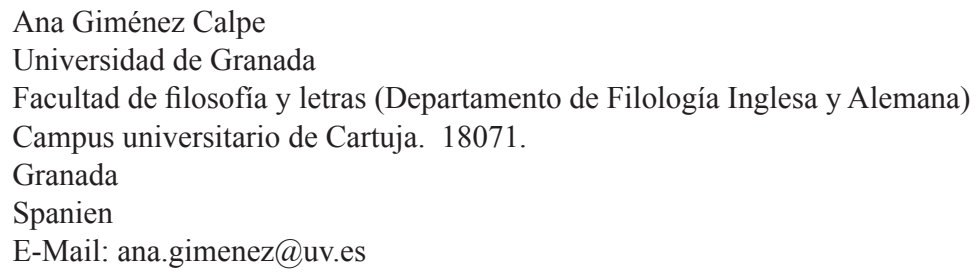

